\title{
More Harm than Good?
}

\author{
Jennifer L. Gnerlich, MD and Mitchell C. Posner, MD \\ Department of Surgery, University of Chicago, Chicago, Illinois
}

Despite all the research-driven "breakthroughs" in the management of pancreatic cancer, the one constant for patients to have any chance at achieving long-term survival is that they are able to undergo resection with curative intent. It is also evident from data generated from phase III randomized trials that chemotherapy (with or without radiation) delivered in the adjuvant setting improves survival in this highly lethal cancer. Surgery followed by chemotherapy or chemoradiotherapy is considered the standard of care for most patients. Yet, considering the extent of surgery necessary to achieve a negative margin resection for cancer of the head of the pancreas, initiating or completing this standard treatment sequence is unfortunately not straightforward. Pancreaticoduodenectomy (PD) is known to be associated with one of the highest postoperative complication rates, up to and exceeding $50 \%$ in many series, even in the most experienced surgical hands at high-volume centers. So, this begs the question: what is the impact of surgical complications on the receipt and timing of adjuvant therapy post-PD?

In an attempt to answer this very question, $\mathrm{Wu}$ et al. have conducted a comprehensive, retrospective review of their extensive experience with PD for adenocarcinoma of the pancreas at Johns Hopkins to analyze the impact of postoperative complications on the receipt of adjuvant therapy, both in the timing and type of treatment. The authors hypothesize that delay in the delivery of adjuvant therapy secondary to postoperative complications would abrogate the potential benefit the patient derives from multimodality

This is an invited editorial for the article available at doi:10.1245/ s10434-014-3722-6

\section{(C) Society of Surgical Oncology 2014}

First Received: 1 April 2014;

Published Online: 15 July 2014

M. C. Posner, MD

e-mail: mposner@surgery.bsd.uchicago.edu therapy and subsequently decrease survival. Although this is a reasonable hypothesis, one needs to further dissect the data presented to determine whether it is supportive of this premise. The authors demonstrate that the presence of a complication was associated with a significant delay in time to adjuvant therapy, but the grade of complication was not. Despite having an overall complication rate of $49.1 \%$, clinically severe complications $(\geq \mathrm{IIIb})$ occurred in only $4.2 \%$ of their patients. Clearly, the number of severe complications is too small for a meaningful analysis of severe morbidity, and this may not be as clinically relevant as the patients who had less severe complications $(<\mathrm{III} b)$. From a clinically relevant standpoint, the patients with these severe complications may never recover to a level that will allow them to ever undergo adjuvant therapy; therefore, the complications we should be focusing on are the less severe complications. ${ }^{1}$ It is also likely that a low-severity complication, such as a wound infection, could cause treatment to be significantly prolonged as compared with delayed gastric emptying, but the Clavien-Dindo score is not descriptive enough to decipher these relative differences in their grading system. Thus, it may behoove investigators to find a different way to analyze the complications that will be particularly clinically relevant after a PD.

In this review, only $54.3 \%$ of the patients received postoperative therapy, with $41.3 \%$ receiving multimodality adjuvant therapy. On multivariate analysis, age $>68$ years and length of stay $>9$ days were associated with a decreased chance of receiving adjuvant therapy. Postoperative complications (both presence and grade) were shown to reduce the intensity of the adjuvant therapy, with patients more likely to receive single-agent chemotherapy or radiation therapy if they had a postoperative complication than if they did not. Here again the numbers are too small to separate out single-agent chemotherapy from radiation treatment alone, and our focus is on multimodality therapy. As expected, receipt of multimodality therapy correlated with a significantly longer median 
survival. Perhaps more important than type of therapy or time to delivery of adjuvant therapy is whether patients actually completed the planned cycles of chemotherapy. Unfortunately, the authors did not provide complete information regarding the number of patients who completed the full course of intended treatment. Recent evidence suggests that completion of all planned cycles of adjuvant therapy, rather than early initiation of chemotherapy, is an independent prognostic factor and improves survival. $^{2}$ Thus, the type of therapy and completion of therapy may be more important than the actual timing of adjuvant treatment in affecting the long-term outcomes and survival of patients.

Despite these few shortcomings, the authors provide us with reasonable and expected conclusions. For patients who did not receive adjuvant therapy, those who experienced complications had a significantly decreased survival compared with those without complications (10.7 vs. 15.7 months). Overall, patients who received adjuvant therapy had improved survival when compared with patients who did not receive any adjuvant therapy. However, the more compelling finding was that the patients who received adjuvant therapy had a similar survival whether they incurred postoperative complications or not (20.4 vs. 22.5 months, respectively; $p>0.05$ ). This once again suggests that the actual receipt of adjuvant therapy, and not necessarily the timing, is the more clinically relevant factor to be attentive to after resection of pancreatic cancer.

Finally, this article raises the question: when does the delay in therapy equate to receiving no therapy at all? The overall median time to adjuvant therapy was 60 days in this study and is fairly consistent with other reports looking at postoperative complications and the receipt of adjuvant therapy after PD. ${ }^{1}$ Predictably, the presence of a postoperative complication significantly delayed the median time to adjuvant therapy, although we agree with the authors that a delay of 7 days is of questionable clinical significance (62 vs. 55 days). With the omission of adjuvant therapy, and not time delay to adjuvant treatment, having such a strong correlation with survival, it is natural to ask: is there a time point where delay of adjuvant therapy becomes equivalent to not receiving adjuvant therapy at all? More importantly, when is that time point and, when it is reached, should we not offer postoperative treatment? Early initiation of chemotherapy may be associated with improved survival for colorectal and breast cancers, but the complication rate and recovery period from curative intent procedures in these malignancies are much different than from PD. Time to recover from an immune and nutritional standpoint, not just from postoperative complications, may take longer after a PD than from a colectomy or mastectomy, thus possibly providing patients with a longer window to treat postoperatively. The confounding issue with pancreatic cancer is not knowing the exact length of time after a PD that is outside of the therapeutic window for adjuvant therapy to confer a potential survival benefit.

In summary, this study reminds us that postoperative complications are common after PD. The presence of a complication delays time to adjuvant therapy, but time to adjuvant therapy was not associated with survival. More importantly, postoperative complications reduce the likelihood of receiving multimodality therapy, which does correlate with a worse survival. We are also reminded that only half of the patients are receiving the prescribed standard of care adjuvant therapy, and omission of adjuvant therapy is clearly associated with diminished survival whether a patient has a complication or not. Since highergrade complications and severe complications were both significantly associated with not receiving adjuvant therapy, the authors conclude that identifying patients at risk for complications who would be unlikely to receive adjuvant therapy would be beneficial. This continues to be a very difficult task, because the clinical and biological factors remain ambiguous.

Yet, there is support for their assertion that these patients may benefit from a neoadjuvant approach. Tzeng et al. demonstrate in their study that patients receiving neoadjuvant treatment compared with a surgery-first approach were more likely to complete their multimodality treatment and have better survival. ${ }^{3}$ In addition, rates of postoperative complications were the same between those two groups. So, if the goal is to truly not do more harm than good after PD, we need to focus on how to deliver the full course of multimodality therapy to pancreatic cancer patients in order to improve their outcome and survival. Future studies focusing on the neoadjuvant approach seem to be a good step in this direction, especially if completion of the planned course of chemotherapy is more likely to happen in the preoperative setting without delaying the time needed to undergo surgery. In fact, the current trend favors the preoperative paradigm, as evidenced by the approximately 30 trials nationwide that are actively accruing patients to studies exploring neoadjuvant therapy approaches with either resectable or borderline resectable pancreatic cancer. ${ }^{4}$ Investigating new ways to ultimately improve on the survival rates seen with pancreatic cancer while balancing the morbidity of PDs is not just a theoretical treatment option but has now become reality.

\section{REFERENCES}

1. Merkow RP, Bilimoria KY, Tomlinson JS, et al. Postoperative complications reduce adjuvant chemotherapy use in resectable pancreatic cancer. Ann Surg. 2013;00:1-6.

2. Valle JW, Palmer D, Jackson R, et al. Optimal duration and timing of adjuvant chemotherapy after definitive surgery of ductal 
adenocarcinoma of the pancreas: ongoing lessons from the ESPAC-3 study. J Clin Oncol. 2014;32:504-12.

3. Tzeng CD, Tran Cao HS, Lee JE, et al. Treatment sequencing for resectable pancreatic cancer: influence of early metastases and surgical complications on multimodality therapy completion and survival. J Gastrointest Surg. 2014;18:16-25.

4. ClinicalTrials.gov. http://www.clincaltrials.gov. Accessed 25 Mar 2014. 